\title{
Novel bone grafting technique in stand-alone ALIF procedure combining allograft and autograft ('Northumbria Technique')—Fusion rate and functional outcomes in 100 consecutive patients
}

\author{
Ata George Kasis ${ }^{1} \cdot$ Cyrus Jensen $^{1} \cdot$ Rahul Dharmadhikari $^{1} \cdot$ Benjamin Ross Emmerson ${ }^{1} \cdot$ Matthew Mawdsley $^{1}$
}

Received: 25 August 2020 / Revised: 3 January 2021 / Accepted: 27 January 2021 / Published online: 15 February 2021

(c) The Author(s) 2021

\begin{abstract}
Introduction Successful ALIF surgery depends upon achieving solid fusion, whilst avoiding significant complications. Herein, we present the 'Northumbria Technique' of combining allograft with autograft in order to achieve solid interbody fusion.

Materials and Methods A single-surgeon series of 100 consecutive patients undergoing stand-alone ALIF from 2016 to 2019 was studied. All had percutaneously harvested iliac crest bone graft (ICBG) dowels inserted into blocks of fresh frozen femoral head (FFFH) allograft, which were then inserted into the ALIF cages. Patients had dynamic radiographs at 4 months, $\mathrm{CT}$ at 6 months, and patient reported outcome measure scores (PROMS) throughout.

Results One hundred patients (average age 44.8 years) were followed-up for an average of 29.1 months. Ninety-four (94\%) patients were assessed as having fused on both CT and radiographs by an independent Radiologist. Three (3\%) patients had abolition of movement on radiographs, but either lacked a CT scan or failed to meet Williams criteria for fusion. Two patients failed to attend for any imaging, so were considered not fused, and one patient had no evidence of fusion in either modality. There was a significant improvement in all PROMS. There were no intra-operative complications, and one patient had transient donor-site pain.

Conclusions The newly described 'Northumbria Technique' utilises the osteoconductive characteristics of the FFFH allograft, as well as the osteoinductive and osteogenic properties of the ICBG autograft. It gives high fusion rates (94-97\%) and statistically significant improvements in PROMS, whilst avoiding the complications of harvesting a large amount of autograft and the huge costs of using synthetic agents.
\end{abstract}

Keywords ALIF $\cdot$ Interbody fusion $\cdot$ ICBG $\cdot$ Allograft $\cdot$ Autograft

\section{Introduction}

Successful Anterior Lumbar Interbody Fusion (ALIF) surgery depends upon achieving a solid fusion, whilst avoiding significant complications. ALIF places the bone graft under compression with the largest possible surface area for union and with a uniform load transfer. The reported fusion rate in ALIF varies from 52 [1] to 100\% [2], and this depends on the indication for surgery, the type of bone graft used, and the method used in detecting the fusion (static plain

Ata George Kasis

atakasis@aol.com

1 Wansbeck General Hospital, Woodhorn Ln, Ashington NE63 9JJ, UK radiographs alone versus computerised tomography (CT) scan alone versus combined dynamic radiographs and $\mathrm{CT}$ scan).

Recombinant Human Bone Morphogenic Protein-2 (rhBMP-2), INFUSE Bone Graft (Medtronic Inc, Memphis, $\mathrm{TN})$ has been commonly used to increase fusion rates [2, 3]. Prior to 2016, rhBMP-2 was used in our unit in combination with fresh frozen femoral head (FFFH) allograft in ALIF procedures with excellent results [4] (Fig. 1a). Due to the significant shortage of rhBMP-2 in Europe at the beginning of 2016, a new technique was developed to replace the rhBMP-2 with a core of iliac crest bone graft (ICBG) obtained through a minimally invasive technique.

Herein, we present the 'Northumbria Technique' of combining the FFFH allograft with autograft in one hundred consecutive patients. All had percutaneously harvested 


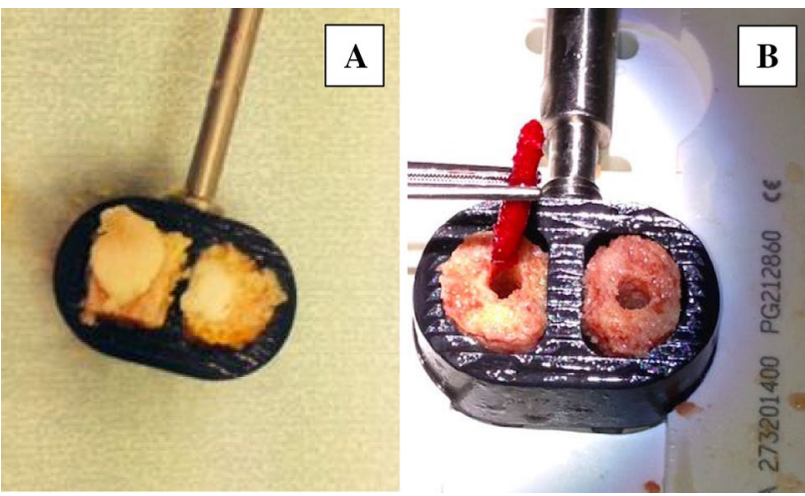

Fig. 1 a Combining rhBMP-2 and FFFH. b Combining ICBG and FFFH

autograft dowels (ICBG) inserted into blocks of FFFH allograft, which were then inserted into the ALIF cages. To our knowledge, this is the first study to report this minimally invasive technique of obtaining autograft in ALIF and also this technique of combining the autograft with the FFFH.

\section{Materials and Methods}

A retrospective review of data which was prospectively collected on one hundred consecutive patients who underwent stand-alone ALIF procedures at L3-S1 (i.e. without posterior instrumentation) between March 2016 and August 2019. The indications for surgery in these patients were neuroforaminal stenosis, recurrent disc prolapse, subtle (grade I) spondylolisthesis, and large central disc prolapse. Contraindications for stand-alone ALIF surgery in our practice included tumours, active infection, significant instability, obesity, extensive retroperitoneal surgery, and pregnancy. All patients had pre- and post-operative patient reported outcome measure scores (PROMS), including visual analogue scores (VAS) for back and leg pain, Oswestry Disability Index (ODI) for low back pain questionnaires and EuroQol scores (EQ-5D) at 6 weeks, 4 months, 8 months and yearly thereafter post-operatively. The student paired $t$ test was used to compare the changes from the baseline with significance defined as $p<0.05$.

The bone graft site was checked at every follow-up encounter, and patients were asked specifically about pain or any other issues related to the donor site. The length of surgery, blood loss, and any intra- or post-operative complications were recorded. The type of cage and plate used was also recorded. At 4 months follow-up, patients had flexion/extension x-rays centred on the fused level to check for movement at this level by measuring the Cobb angle, with less than 2 degrees difference being considered to represent fusion with no movement (Simmons method [5]). Also, the anterior disc heights were measured with $<1 \mathrm{~mm}$ difference between flexion and extension being considered to represent fusion. At 5-6 months post-operatively, patients had a thin-slice $(1 \mathrm{~mm}) \mathrm{CT}$ scan using sagittal and coronal reconstructions to assess bony union (Williams criteria [6]) and this was independently assessed by a fellowship-trained Consultant musculoskeletal radiologist $(\mathrm{RD})$ who was blinded to the $\mathrm{x}$-ray findings. Complete fusion on CT scan was confirmed if the following criteria were met: clearly visible continuous trabecular bone connecting the vertebral bodies, continuous bridging bone surrounding or within the cage, lack of lucency at cage margins, lack of visible fractures of cage or vertebrae, lack of cystic changes within adjacent endplates, lack of linear defects through the new bone formation, and lack of subsidence or dislocation.

Fusion in this trial was only confirmed when both the following criteria had been met: fusion was seen on the CT scan and no movements on the flexion/extension x-rays.

\section{Surgical and bone grafting technique}

The ALIF procedure (including the approach) was performed by a single fellowship-trained Consultant orthopaedic spine surgeon (AK) through a retroperitoneal approach after splitting the rectus sheath longitudinally. The abdominal contents and left ureter were held using an Integra Omni-Tract ${ }^{\circledR}$ retractor (Omni, St. Paul, MN). AUS ${ }^{\mathrm{TM}}$ smooth pins (Steinmann type pins) were used to hold the vessels after mobilisation while performing the procedure. Two blocks of cancellous allograft from a donated FFFH were cut to the size of the graft windows using an oscillating saw.

Prior to March 2016, we adopted the technique described by the Gold Coast Spine Unit [7, 8] where rhBMP-2 sponges were inserted into pre-drilled $3.2 \mathrm{~mm}$ channels inside the FFFH allograft (Fig. 1a). This technique was then modified due to the lack of availability of rhBMP-2, by inserting a core of autograft into the drilled channels of each block of FFFH (Fig. 1b). The autograft cores were obtained by inserting a 10 gauge vertebral needle (Jamshidi Needle $®$, Stryker) into the iliac crest through a $1 \mathrm{~mm}$ puncture wound 3-4 $\mathrm{cm}$ posterior to the anterior superior iliac spine (ASIS) (Fig. 2a). The Jamshidi Needle ${ }^{\circledR}$ was advanced using a mallet until the cortex was breached, at a 30 degree caudal angle and 45 degree angle to the sagittal plane (Fig. 2b,c). The inner insert was then removed, and the needle was tapped and rotated inside the cancellous part of the iliac crest, and then withdrawn while using a syringe to apply negative pressure (Fig. 3). After inserting the cage, an Agesis plate $₫$ (DepuySynthes) was used in all patients, and the screws inserted obliquely through the vertebral rim. 

needle $3-4 \mathrm{~cm}$ posterior to ASIS. b, c Trajectory of needle in iliac crest demonstrated on model pelvis
Fig. 2 a Entry point of Jamshidi
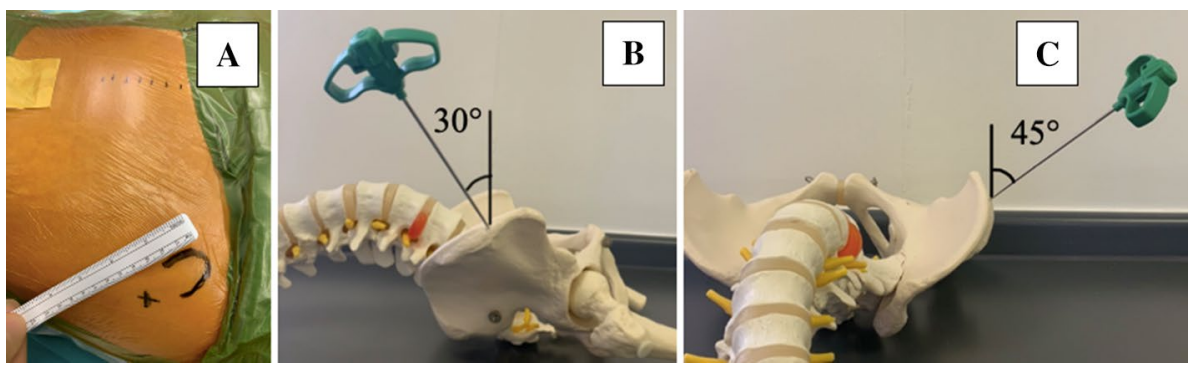

Fig. 3 Stages of taking the bone graft through a minimally invasive technique. a Measuring 3-4 cm posterior to the ASIS. b The Jamshidi Needle ${ }^{\circledR}$ is tapped until the cortex is breached. c The inner insert is removed, and the needle is tapped and rotated inside the iliac crest. d The needle is withdrawn while using a syringe to apply negative pressure. $\mathbf{e}$ The inner insert is used to push the ICBG out of the needle

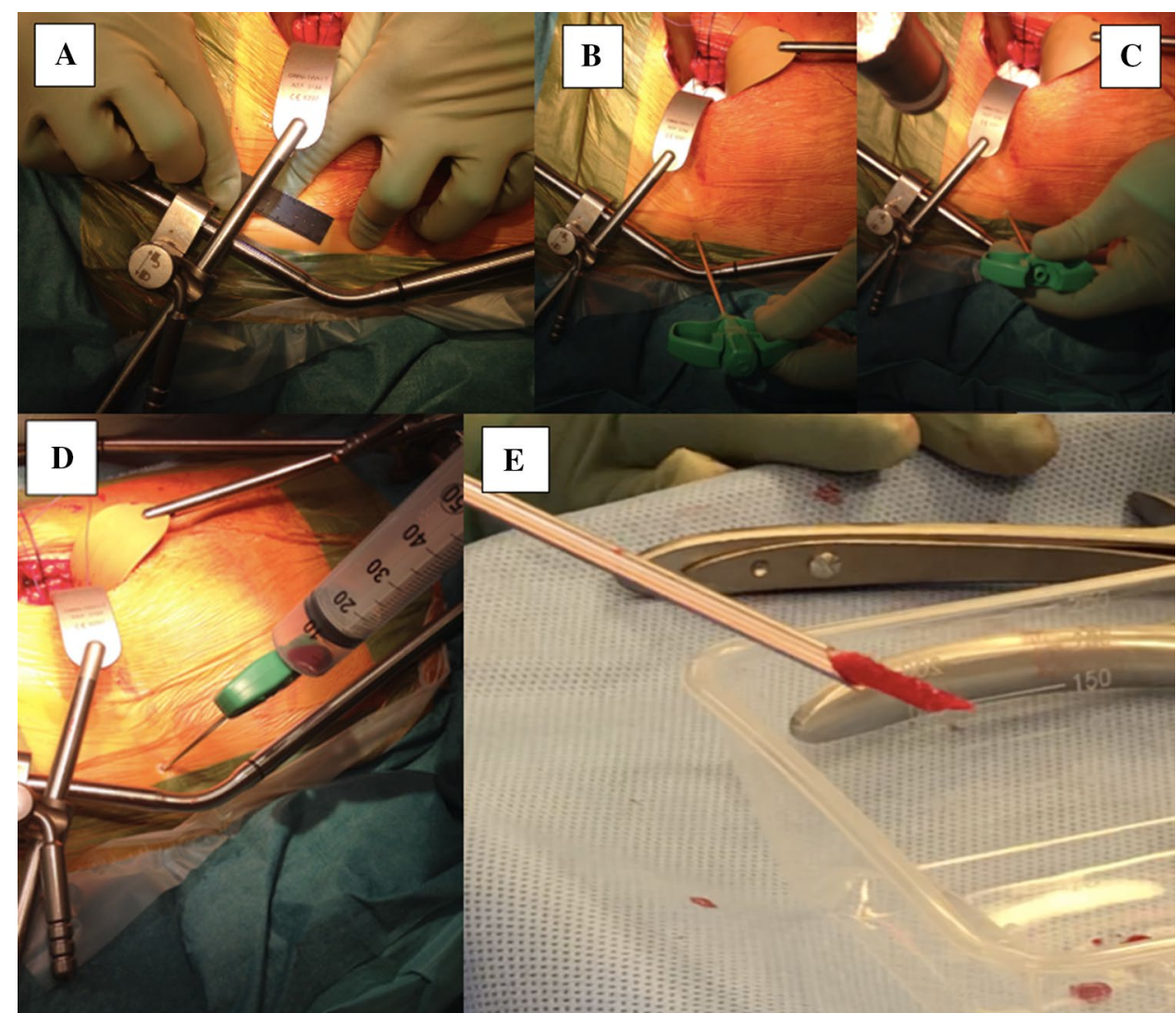

\section{Results}

One hundred consecutive patients were included in the trial, with 108 levels addressed with surgery. Ninety-two patients had surgery at one level (55 at L5/S1, 36 at L4/L5 and one at L3/L4) and eight had two-level ALIF surgery (7 at L4/L5 and L5/S1, and 1 at L3/L4 and L4/L5). Three patients failed to attend for their CT scan $(97 \%$ complete follow-up rate), and the average length of follow-up was 29.1 months (7.9-49.9). The average age at surgery was 44.8 years $(30.2-74.1)$, and there were 63 females and 37 males. The indications for surgery were neuroforaminal stenosis in 40 patients, central stenosis due to a large central disc prolapse with significant loss of disc height and lordosis in 29 patients, and recurrent disc prolapse in 31 patients. A Brantigan cage ${ }^{\circledR}$ (Depuy-Synthes) was used in 86 patients, Continental cage ${ }^{\circledR}$ Globus in 10 , and Australis ${ }^{\circledR}$ (Prism Surgical) ALIF cage in 4. All patients had an Agesis ${ }^{\circledR}($ Depuy-Synthes) plate with 4 screws. Blood loss was less than $150 \mathrm{ml}$ in all patients. The average time for surgery was $74 \mathrm{~min}$ per level (60-130 min).

With three patients failing to attend for post-operative CT scans and a further three patients not fulfilling the CT criteria for fusion [6], the overall rate of complete fusion in this series was $94 \%$. One of the 100 patients (1\%) complained of pain at the bone graft site which settled completely at 8 weeks. At the donor site, there were no infections, neural injuries or haematomas. There was a statistically significant improvement in the average VAS for leg pain from 6.8 preoperatively to 2.1 at two years $(p<0.001)$. Similarly, there was a statistically significant improvement in VAS for back 
pain from 7.2 pre-operatively to 2.5 at two years $(p<0.001)$. There was a statistically significant improvement in ODI from 49.4 pre-operatively to 19 at two years $(p<0.001)$. Similarly, EQ-5D improved from 65 pre-operatively to 84 at two years $(p<0.001)$ (Fig. 4).

\section{Discussion}

The overall aims of ALIF surgery are radical discectomy and restoration of neuroforaminal height with direct and/or indirect decompression of neural structures, solid interbody fusion with abolition of motion, and restoration of normal lumbar sagittal profile. These factors should, in turn, lead to improvements in the patients' function and clinical symptomatology. As with previous studies [9-11], we have shown a case series of 100 patients where ALIF surgery led to significant improvements in leg and back pain (VAS), global disability (EQ-5D), and functional (ODI) PROMS.

The 'Northumbria Technique' of inserting percutaneously harvested ICBG into FFFH allograft in the graft windows was a development by our team driven by the sudden lack of availability of rhBMP-2. We hoped the new technique would maintain the previously described high ALIF fusion rates [4] achieved with allograft and rhBMP-2, whilst reducing the costs and without adding the complications of donor site morbidity seen in previous trials using ICBG [12-15]. Removing the need for rhBMP-2 would also potentially reduce the risk of BMP-related complications such as radiculitis [16]. The combination of FFFH and ICBG contributes to the stability of the construct (structural bone graft and cage) as it acts as load-bearing and load-sharing, adding a mechanical advantage helping to achieve good quality bony fusion.

Non-union following spine fusion is multifactorial and negatively affects patient outcomes $[1,11]$. The reported rate of spinal fusion varies depending on the radiological method used for assessing the fusion. Lower fusion rates were reported using fine cut CT scan compared to plain radiographs $[17,18]$. Our use of both $\mathrm{CT}$ and dynamic radiographs to confirm fusion should result in a more reliable evaluation.

Using the CT-based Williams criteria [6], we found 94 of the 97 patients who attended for a scan had fused. (Fig. 5). All of these 94 CT-confirmed fusion patients also had abolition of movement on dynamic radiographs, using both the validated Simmons method of measuring Cobb angle changes, and the unvalidated anterior disc height change method. Notably, two of the three patients who had radiological non-union on the CT scan had lost segmental movement on the dynamic radiographs and were asymptomatic, suggesting that they were probably also fused. The other patient with non-union on CT had 5.5 degrees of movements on the dynamic $\mathrm{x}$-rays and was symptomatic and so was definitely not united.

In this series, the CT-confirmed fusion rate was $94 \%$ (we considered the three patients who did not attend for their CT scan to have not fused). A further $3 \%$ were felt to have successfully fused by 4 months based on a lack of movement on dynamic radiographs using the Simmons method and measuring the anterior disc height, as well as being asymptomatic. The patients who had non-union had a normal BMI.

The literature regarding ALIF surgery is extremely heterogenous, and so comparisons are not easily made when studying fusion rates. Many surgeons regard ICBG autograft as the 'Gold Standard' bone substitute material in spine fusion surgery; however, fusion rates in several ALIF studies where ICBG was used in isolation (Tiusanen et al. [19] 81\%, Christensen et al. [1] 76\%, Cho et al. [20] 86\%) were much lower than in this paper where the 'Northumbria Technique' was used (94-97\%). Pavlov et al. [10] were able to increase the fusion rates by supplementing the ALIF with posterior instrumentation, whilst others were able to achieve high fusion rates by adding in synthetic bone substitutes (Chatha et al. [3] 94\%, Rao et al. [9] 98\% for 1 level, $81.5 \%$ for 2 level ALIF). These were similar fusion rates to our described
Fig. 4 Graphs showing the functional outcomes pre- and post-operatively
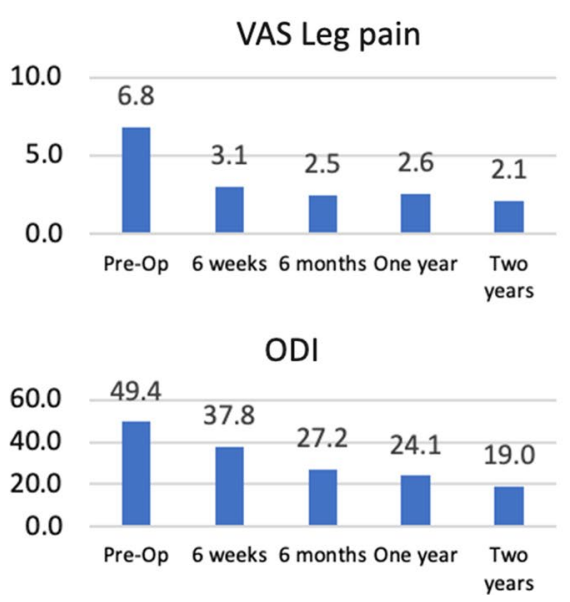

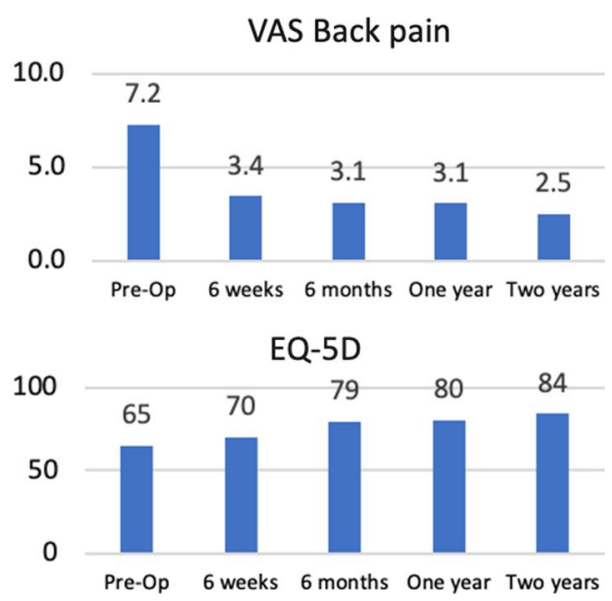




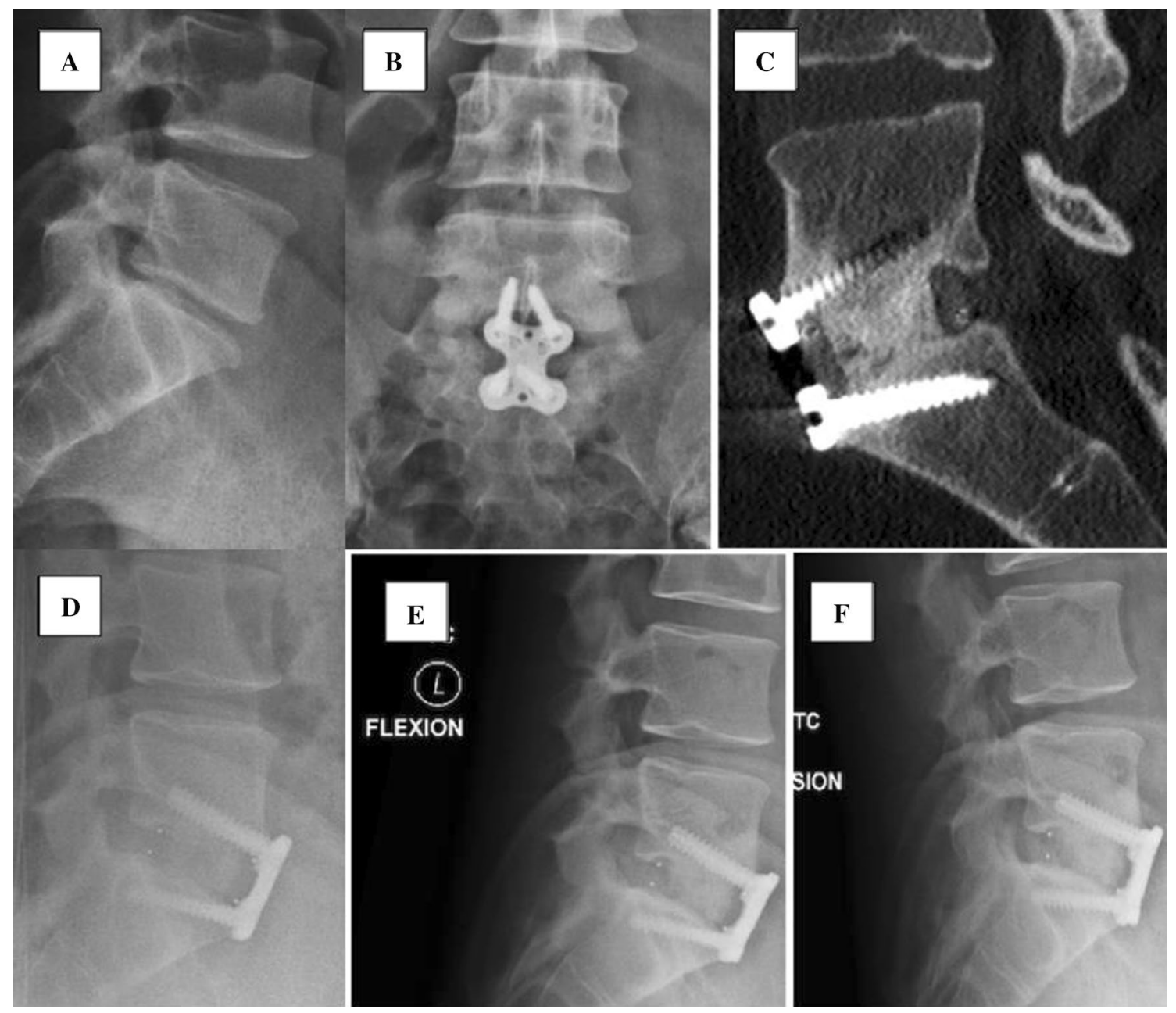

Fig. 547 year old male, who underwent ALIF of L5/S1 for recurrent disc prolapse after having two discectomies with the second one complicated by an incidental durotomy. He presented with back and leg pain. a Standing lateral x-ray shows loss of disc height and lordosis,

stand-alone ALIF technique, but with the added cost and complication risks of posterior surgery.

Strube et al. [17] found a CT-confirmed fusion rate of $70.6 \%$ using only fresh dried allograft in standalone ALIF. The combination of allograft and autograft dowels in ALIF surgery has been previously investigated by Newman et al. [21] who used x-rays only to report a $88.9 \%$ fusion rate in 32 patients. However, using the 'Northumbria Technique' by combining osteoinductive and osteoconductive bone graft, we achieved a higher fusion rate with good functional outcomes.

\section{Complications}

In this study series, there were no intra-operative complications, deep infections or incidences of symptomatic venous thromboembolism. Early complications included b Antero-posterior x-ray after ALIF, c CT scan showing complete fusion, d Standing lateral x-ray after ALIF, e Flexion lateral x-ray, $\mathbf{f}$ Extension lateral $\mathrm{x}$-ray

one superficial wound infection which was treated with oral antibiotics, and one retroperitoneal haematoma which was treated with a blood transfusion. One patient required surgery 40 months later for adjacent segment degeneration, with posterior decompression. There was one patient with a symptomatic non-union in this series of 100 patients, who continued to smoke after their surgery, despite undergoing a pre-operative smoking cessation course and confirming they had stopped smoking - they are currently awaiting revision surgery.

The complication rate of harvesting ICBG in the literature varies from 2.8 to $43 \%$ [12-15], and the complications include infection, seroma, meralgia paraesthetica, haematoma, chronic pain, hernia, additional surgery, and wound dehiscence. In this series, there was only one patient (1\%) who complained of pain at the donor site, which had fully resolved by 8 weeks post-operatively. With no other 
complications reported as a result of harvesting the ICBG cores, this 'Northumbria Technique' seems to offer the benefits of autograft (osteoconductive, osteoinductive, and osteogenic) without the morbidity associated with harvesting autograft.

\section{Cost}

Prior to this series of patients, our standard practice included filling the allograft drill channels with rhBMP-2-impregnated dowels. The costs of rhBMP-2 vary throughout the world healthcare systems. In our hospital, before the 2016 shortages, the cost of a large dose of rhBMP-2 (INFUSE) was $£ 1750$. Using the 'Northumbria Technique', this cost is substituted with the cost of the single-use Jamshidi needle ${ }^{\circledR} £ 55$ each (cost to our hospital). Extrapolating this over the 100 cases in this series, this represents a cost saving of $£ 169,500$, whilst delivering similar fusion and complication rates [4]. It is accepted that this study was not set up to compare costs, fusion or complication rates between two techniques; however, it seems reasonable to conclude that the 'Northumbria Technique' does reduce the costs of standalone ALIF surgery compared to using rhBMP-2. However, this argument does not take into account the cost of FFFH.

\section{Limitations}

The main limitation to this trial is its retrospective study design, with the uncontrolled biases that this can bring. It is, however, a large consecutive single-surgeon series, with similar results to a previous series from this unit [4], and a high follow-up rate (97\%) over an average of 29.1 months. Due to the lack of a control group (with just FFFH in the cage), it is not possible to truly ascertain the impact of the 'Northumbria Technique' of inserting ICBG dowels through the FFFH; other than to conclude that it gives a better fusion rate and a lower complication rate than previous studies investigating just allograft in stand-alone ALIF [17, 22, 23]. Undoubtedly, the high-volume ALIF experience of the author (AK) will have had an impact on the high fusion rate and low complication rate, and it remains to be seen if these rates can be reproduced by other surgeons utilising the 'Northumbria Technique'.

\section{Summary}

Obtaining a solid fusion is of paramount importance when trying to achieve good clinical outcomes following standalone ALIF spine surgery. The newly described 'Northumbria Technique' involves harvesting autologous ICBG dowel cores and inserting them into pre-drilled channels in FFFH allograft, which sit in the interbody cage graft windows. It has been shown to give high fusion rates (94-97\%) and statistically significant improvements in PROMS, whilst avoiding the complications of harvesting a large amount of autograft and the cost of using synthetic agents such as rhBMP-2.

Given the potential for huge cost savings to healthcare providers, it is recommended that further prospective randomised controlled research is conducted comparing the outcomes and costs using a variety of different bone substitutes, including the 'Northumbria Technique', in spinal fusion surgery.

\section{Compliance with ethical standards}

Conflict of interest None of the authors has any potential conflict of interest.

Open Access This article is licensed under a Creative Commons Attribution 4.0 International License, which permits use, sharing, adaptation, distribution and reproduction in any medium or format, as long as you give appropriate credit to the original author(s) and the source, provide a link to the Creative Commons licence, and indicate if changes were made. The images or other third party material in this article are included in the article's Creative Commons licence, unless indicated otherwise in a credit line to the material. If material is not included in the article's Creative Commons licence and your intended use is not permitted by statutory regulation or exceeds the permitted use, you will need to obtain permission directly from the copyright holder. To view a copy of this licence, visit http://creativecommons.org/licenses/by/4.0/.

\section{References}

1. Christensen FB, Karlsmose B, Hansen ES, Bünger CE (1996) Radiological and functional outcome after anterior lumbar interbody spinal fusion. Eur Spine J. https://doi.org/10.1007/BF003 04343

2. Kleeman TJ, Michael Ahn U, Talbot-Kleeman A (2001) Laparoscopic anterior lumbar interbody fusion with rhBMP-2: A prospective study of clinical and radiographic outcomes. Spine (Phila Pa 1976). https://doi.org/10.1097/00007632-200112150-00026

3. Chatha G, Foo SWL, Lind CRP et al (2014) Anterior lumbar interbody surgery for spondylosis results from a classicallytrained neurosurgeon. J Clin Neurosci. https://doi.org/10.1016/j. jocn.2013.11.033

4. Kasis AG (2019) Stand-Alone Interbody Devices: Static Versus Dynamic. In: Handbook of Spine Technology

5. Simmons JW (1985) Posterior lumbar interbody fusion with posterior elements as chip grafts. Clin Orthop Relat Res. https://doi. org/10.1097/00003086-198503000-00011

6. Williams AL, Gornet MF, Burkus JK (2005) CT evaluation of lumbar interbody fusion: Current concepts. Am. J, Neuroradiol

7. Scott-Young M, McEntee L, Schram B et al (2017) The concurrent use of lumbar total disc arthroplasty and anterior lumbar interbody fusion. Spine (Phila Pa 1976). https://doi.org/10.1097/BRS.00000 00000002263

8. Scott-Young MN, Zotti MGT (2019) Allograft Use in Modern Spinal Surgery. In: Handbook of Spine Technology

9. Rao PJ, Loganathan A, Yeung V, Mobbs RJ (2015) Outcomes of anterior lumbar interbody fusion surgery based on indication. 
Neurosurgery 76:7-24. https://doi.org/10.1227/NEU.0000000000 000561

10. Pavlov PW, Meijers H, Van Limbeek J et al (2004) Good outcome and restoration of lordosis after anterior lumbar interbody fusion with additional posterior fixation. Spine (Phila Pa 1976). https:// doi.org/10.1097/01.brs.0000137067.68630.70

11. Riouallon G, Lachaniette CHF, Poignard A, Allain J (2013) Outcomes of anterior lumbar interbody fusion in low-grade isthmic spondylolisthesis in adults: a continuous series of 65 cases with an average follow-up of 6.6years. Orthop Traumatol Surg Res. https ://doi.org/10.1016/j.otsr.2012.12.009

12. Arrington ED, Smith WJ, Chambers HG et al (1996) Complications of iliac crest bone graft harvesting. Clin Orthop Relat Res. https://doi.org/10.1097/00003086-199608000-00037

13. Banwart J, Asher M (1995) Iliac crest bone graft harvest donor site morbidity: a statistical evaluation. Spine (Phila Pa 1976). https:// doi.org/10.1097/00007632-199505000-00012

14. Silber JS, Anderson DG, Daffner SD et al (2003) Donor site morbidity after anterior iliac crest bone harvest for single-level anterior cervical discectomy and fusion. Spine (Phila Pa 1976). https ://doi.org/10.1097/00007632-200301150-00008

15. Schnee CL, Freese A, Weil RJ, Marcotte PJ (1997) Analysis of harvest morbidity and radiographic outcome using autograft for anterior cervical fusion. Spine (Phila Pa 1976). https://doi. org/10.1097/00007632-199710010-00005

16. Lubelski D, Abdullah KG, Steinmetz MP et al (2015) Adverse events with the use of rhBMP-2 in thoracolumbar and lumbar spine fusions: a 9-year institutional analysis. J Spinal Disord Tech. https://doi.org/10.1097/BSD.0b013e318287f2e2

17. Strube P, Hoff E, Hartwig T et al (2012) Stand-alone anterior versus anteroposterior lumbar interbody single-level fusion after a mean follow-up of 41 months. J Spinal Disord Tech. https://doi. org/10.1097/BSD.0b013e3182263d91

18. Hoff E, Strube P, Gross C et al (2010) Monosegmental anterior lumbar interbody fusion with the SynFix-LR device. A prospective 2-year follow-up study. Orthopade. https://doi.org/10.1007/ s00132-010-1654-9

19. Tiusanen H, Seitsalo S, Österman K, Soini J (1996) Anterior interbody lumbar fusion in severe low back pain. Clin Orthop Relat Res. https://doi.org/10.1097/00003086-199603000-00018

20. Cho CB, Ryu KS, Park CK (2010) Anterior lumbar interbody fusion with stand-alone interbody cage in treatment of lumbar intervertebral foraminal stenosis: Comparative study of two different types of cages. J Korean Neurosurg Soc 47:352-357. https ://doi.org/10.3340/jkns.2010.47.5.352

21. Newman MH, Grinstead GL (1992) Anterior lumbar interbody fusion for internal disc disruption. Spine (Phila Pa 1976) 17:831833. https://doi.org/10.1097/00007632-199207000-00017

22. Shim JH, Park CK (2011) Erratum: Comparison of instrumented posterolateral fusion versus percutaneous pedicle screw fixation combined with anterior lumbar interbody fusion in elderly patients with L5-S1 isthmic spondylolisthesis and foraminal stenosis. J Neurosurg Spine. https://doi.org/10.3171/2011.5.spine10653a

23. Hacker RJ (1997) Comparison of interbody fusion approaches for disabling low back pain. Spine (Phila Pa 1976) 22:660-666. https ://doi.org/10.1097/00007632-199703150-00017

Publisher's Note Springer Nature remains neutral with regard to jurisdictional claims in published maps and institutional affiliations. 